\title{
AN INTRINSIC METRIC FOR PARTS
}

\section{H. S. BEAR AND MAX L. WEISS ${ }^{1}$}

1. Introduction. The concept of a "Gleason part" has been defined in several settings of increasing generality. The original part introduced by Gleason [4] was a subset of the spectrum of a function algebra. This was extended in [1] to the state space, or carrier, of a linear space of real functions. Recently, Lewittes has extended the idea of part to the unit ball of the adjoint $E^{*}$, for any normed space $E$ [7]. The spectrum of a function algebra and the state space for a function space are both subsets of the ball of the adjoint space, and Lewittes' definition includes the preceding ones. Each of the three notions of part is associated with a metric defined on each part. In this paper we will show how parts can be defined most naturally in any convex set which contains no line. Our definition is an extension of the geometric characterization of [1]. We then define a metric for each part in terms of the geometry, and without reference to any topology or any space of functions. We show that this intrinsic metric is topologically equivalent to each of the previously defined metrics in the cases in which the latter are defined. For a compact convex set $K$ in a locally convex linear topological space $E$, we also show that two points are in the same part of $K$ if and only if they have representing measures whose Radon-Nikodym derivatives with respect to each other are bounded. This extends Bishop's characterization of part [2] to this general setting.

The original definition of Gleason part for a function algebra $A$ can be recaptured as follows. The spectrum $S_{A}$ of $A$ consists of the set of nonzero (necessarily continuous) homomorphisms of $A$, with the $w^{*}$ topology it inherits as a subset of $A^{*}$. Take $E$ to be the real restriction of $A^{*}$ (i.e., restrict the scalar multiplication to the reals) with the $w^{*}$ topology and let $K=\left\{F \in A^{*}: F(1)=\|F\|=1\right\}$. Then $K$ is a compact convex set containing $S_{A}$. The Gleason parts of $S_{A}$ are the intersections with $S_{A}$ of the parts of $K$ as defined below [1].

The authors are indebted to Joseph Kist for pointing out an unnecessary restriction in our original condition on $K$.

2. Geometric parts and the intrinsic metric. Let $E$ be a real linear

Presented to the Society, November 25, 1966; received by the editors August 13, 1966.

1 This research was supported in part by Grant NSF-GP-6118 of the National Science Foundation. 
space, and let $K$ be any convex set in $E$ which contains no whole line of $E$. For $x, y \in K$, we define (cf. [1], and [3, p. 85]) $x \sim y$ if and only if $x=y$ or $x$ and $y$ are in some open segment within $K$. A two-dimensional geometric argument shows that $\sim$ is an equivalence relation on $K$. The equivalence classes of $\sim$ will be called the parts of $K$.

Our definition of a metric in each part depends on the following geometric lemma. If $x, y \in K$, we denote by $[x, y]$ the line segment joining $x$ and $y$. We say that $[x, y]$ extends by $\epsilon$ if $\epsilon>0$ and $x+\epsilon(x-y)$, $y+\epsilon(y-x)$ are both in $K$. Clearly $x \sim y$ if and only if $[x, y]$ extends by some $\epsilon$.

Lемма 1. Let $K$ be a convex set which contains no line and $x, y, z \in K$. If $[x, z]$ extends by $a$ and $[y, z]$ extends by $b$, then $[x, y]$ extends by $c$, where $\left(1+a^{-1}\right)\left(1+b^{-1}\right)=\left(1+c^{-1}\right)$.

Proof. Let $c$ be defined by the formula given above, and let $\lambda=(1+b) /(1+a+b), \mu=(1+a) /(1+a+b)$. It is easy to verify that

$$
\begin{aligned}
& x+c(x-y)=\lambda[x+a(x-z)]+(1-\lambda)[z+b(z-y)], \\
& y+c(y-x)=\mu[y+b(y-z)]+(1-\mu)[z+b(z-x)] .
\end{aligned}
$$

Since the points $x+c(x-y), y+c(y-x)$ are convex combinations of points which are in $K$ by hypothesis, $[x, y]$ extends by $c$.

Now define a function $d(x, y)$, for $x \sim y$, as follows: $d(x, x)=0$ and for $x \neq y$

$$
d(x, y)=\inf \left\{\log \left(1+\epsilon^{-1}\right):[x, y] \text { extends by } \epsilon\right\} .
$$

Lemma 2. $d$ is a metric on each part of $K$.

Proof. Clearly $d(x, y) \geqq 0$ and $d$ is symmetric. If $x \sim y$ and $x \neq y$, then $[x, y]$ extends by some $\epsilon$, and the set of such $\epsilon$ is bounded. Hence $d(x, y)=0$ if and only if $x=y$. To check the triangle inequality, let $x \sim y \sim z$. If $[x, y]$ extends by $a$ and $[y, z]$ extends by $b$, then from Lemma $1[x, z]$ extends by an amount $c$ which satisfies

$$
\log \left(1+a^{-1}\right)+\log \left(1+b^{-1}\right)=\log \left(1+c^{-1}\right) .
$$

By picking $a$ and $b$ so that the terms on the left approach $d(x, y)$ and $d(y, z)$ we see that

$$
d(x, y)+d(y, z) \geqq d(x, z) .
$$

We will call $d$ the intrinsic metric on the parts of $K$. Clearly $d$ can be extended to $K \times K$ by letting $d(x, y)=\infty$ if $x$ and $y$ are in different parts. By letting $d^{*}=d /(1+d)$, we obtain a metric on $K \times K$ such that $x \sim y$ if and only if $d^{*}(x, y)<1$. 
3. Parts and representing measures. We suppose in this section that $K$ is a compact convex subset of a real locally convex linear topological space $E$. As usual, $E^{*}$ will denote the adjoint space of continuous linear functionals on $E$. The spectrum of a function algebra, and the carrier of a function space are subsets of the unit ball of the adjoint space, and are given the $w^{*}$ topology of the adjoint space. The $w^{*}$ topology on the adjoint makes it a locally convex space, and the unit ball is compact in this topology. The $w^{*}$-continuous linear functionals on $E^{*}$, with the $w^{*}$ topology, correspond (by evaluation) to the elements of $E$.

Let $\Gamma$ be the closure of the set of extreme points of $K$. A representing measure for a point $x \in K$ is a positive Baire probability measure $\mu$ on $\Gamma$ such that $u(x)=\int_{\Gamma} u d \mu$ for all $u \in E^{*}$. Every point $x$ of $K$ is a limit of convex combinations of extreme points by the Kreinn-Mil'man theorem. Each such convex combination can be represented by the corresponding convex combination of unit point masses at these extreme points. The set of probability measures on $\Gamma$ is $w^{*}$ compact, so there will be a measure $\mu_{x}$ which is an accumulation point of the set of convex combinations of point masses. The measure $\mu_{x}$ will be a representing measure for $x$ by linearity. Hence each $x \in K$ has at least one representing measure $\mu_{x}$. Conversely, each positive probability measure $\mu$ on $\Gamma$ is a weak limit of convex combinations of point masses. These convex combinations will represent points of $K$, and since $K$ is compact, the limit $\mu$ represents a point of $K$.

The following theorem characterizes the parts of $K$ both in terms of the representing measures (cf. [2]) and in terms of a Harnack-type inequality (cf. [1]).

Theorem 1. Let $K$ be a compact convex set in a locally convex linear topological space $E$, and $\Gamma$ be the closure of the set of extreme points of $E$. The following conditions are equivalent for all $x, y \in K$ and all $\epsilon>0$.

(i) $[x, y]$ extends by $\epsilon$.

(ii) There are representing measures $\mu_{x}, \mu_{y}$ on $\Gamma$ such that $\mu_{x} \leqq\left(1+\epsilon^{-1}\right) \mu_{y}$ and $\mu_{\nu} \leqq\left(1+\epsilon^{-1}\right) \mu_{x}$.

(iii) Whenever $u$ is a continuous affine (linear functional plus a constant) function which is positive on $K,\left(1+\epsilon^{-1}\right)^{-1} \leqq u(x) / u(y) \leqq 1+\epsilon^{-1}$.

Proof. (i) implies (ii): Suppose that $[x, y]$ extends by $\epsilon$, so that the points $x^{\prime}=x+\epsilon(x-y), y^{\prime}=y+\epsilon(y-x)$ are in $K$. Then $x=\lambda x^{\prime}$ $+(1-\lambda) y^{\prime}$ and $y=(1-\lambda) x^{\prime}+\lambda y^{\prime}$, where $\lambda=(1+\epsilon) /(1+2 \epsilon)$. If $\mu, \nu$ are representing measures for $x^{\prime}, y^{\prime}$ respectively, then clearly $\mu_{x}=\lambda \mu$ $+(1-\lambda) \nu$ and $\mu_{\nu}=(1-\lambda) \mu+\lambda \nu$ are representing measures for $x$ and $y$. Also $\mu_{x} \leqq A \mu_{y}$ and $\mu_{y} \leqq A \mu_{x}$ where $A$ is the maximum of $\lambda /(1-\lambda)$ and $(1-\lambda) / \lambda$. Since $\lambda /(1-\lambda)=1+\epsilon^{-1}>1$, we have (ii). 
(ii) implies (i). Assume there are representing measures $\mu_{x}$ and $\mu_{y}$ satisfying (ii). Then $\epsilon\left[\left(1+\epsilon^{-1}\right) \mu_{x}-\mu_{y}\right]$ and $\epsilon\left[\left(1+\epsilon^{-1}\right) \mu_{y}-\mu_{x}\right]$ are positive probability measures and hence represent some points $x^{\prime}, y^{\prime} \in K$. By linearity it follows that $x^{\prime}=x+\epsilon(x-y)$ and $y^{\prime}=y+\epsilon(y-x)$, and hence $[x, y]$ extends by $\epsilon$.

(ii) if and only if (iii). The theorem of [2] is stated for points in the same part of the spectrum of a function algebra. However, the proof of [2] without change shows the existence of measures satisfying the inequalities of (ii) given the condition of (iii). The inequalities of (iii) obviously follow from the existence of the measures as in (ii).

4. Metrics on parts. In this section we will show that the metrics which are associated with the several definitions of part are topologically equivalent.

Since the spectrum of a function algebra $A$ is a subset of $A^{*}$, it automatically has the norm-metric of $A^{*}$ defined on it:

$$
G(x, y)=\sup \{|f(x)-f(y)|: f \in A,\|f\| \leqq 1\} .
$$

Gleason showed that the condition $G(x, y)<2$ defines an equivalence relation on the spectrum of $A$, and these equivalence classes are the original Gleason parts.

In [1] a "part metric" $D$ was defined on each part of the carrier of a function space as follows:

$$
D(x, y)=\log \inf \left\{c: c^{-1} \leqq u(x) / u(y) \leqq c \text {, all } u>0\right\} .
$$

Theorem 1 shows that this definition can be extended to any compact convex set $K$ by using the affine functions, and that the resulting function $D$ will be identical with the intrinsic metric $d$. Note that $d$ depends only on the geometry, and in particular is independent of any topology, whereas $D$ appears $a$ priori to depend on the class of continuous linear functionals.

It was shown in [1] that if the spectrum of a function algebra is considered as a subset of the carrier of the function space of real parts of functions in the algebra, the two definitions of part coincide and $D$ and $G$ yield equivalent metrics on each part of the spectrum. Now we turn to the hyperbolic metric introduced by Lewittes to define parts in ball $E^{*}$.

For complex numbers $a$ and $b$ in the closed unit disc let $\chi$ be the pseudohyperbolic metric: $\chi(a, b)=|(a-b) /(1-\bar{a} b)|$. The function $\chi$ is the hyperbolic tangent of the hyperbolic metric (cf. [5, p. 238]), and since the hyperbolic tangent is subadditive, $\chi$ is again a metric. Since $\chi$ is the absolute value of a fractional linear transformation, $\chi(a, b) \leqq 1$ for all $a, b$ in the unit disc. 
Following [7] we define a metric $\rho$ on $K$ as follows:

$$
\rho(x, y)=\sup \left\{x(u(x), u(y)): u \in E^{*},|u|<1 \text { on } K\right\} .
$$

The metric $\rho$ is equivalent to that introduced by Lewittes in [7] to define the "hyperbolic parts" in the case that $K$ is the unit ball of an adjoint space. Lewittes used the hyperbolic metric on the disc rather than the pseudohyperbolic metric we use here. In the case of a function algebra $A$, the metric $\rho$ is equivalent on each part of the spectrum to the $A^{*}$-norm metric introduced by Gleason. This follows from the fact that $\chi$ is equivalent to the Euclidean metric on the open unit disc.

The following theorem compares Lewittes' metric with the intrinsic metric.

THEOREM 2. If $K$ is a symmetric compact convex subset of a locally convex linear topological space, then the metrics $\rho$ and $d$ are equivalent on each part of $K$. In addition $x, y \in K$ are in the same part if and only if $\rho(x, y)<1$.

The proof of Theorem 2 depends on the following lemma of [7].

Lemma 3. Let $k$ and $\theta$ be numbers such that $0<k<k+\theta<1$, and let $\epsilon=\theta / k(1+k+\theta)$. If $a, b$ are complex numbers in the open unit disc with $\chi(a, b) \leqq k$, and $c$ is any point whose Euclidean distance from the segment $[a, b]$ is less than $\epsilon$, then $|a+c(a-b)|<1$ and $\chi(a, a+c(a-b))$ $\leqq k+\theta$.

In geometric language the lemma says that the segment $[a, b]$ can be fattened by a factor $\epsilon$ which does not depend on the positions of $a$ and $b$, but only on $\chi(a, b)$, and the fattened segment will still contain only points whose $\chi$-distance from $a$ is bounded away from 1 . We use this result only on $[-1,1]$. Here the lemma says that the segment $[a, b] \subset(-1,1)$ can be extended by $\epsilon$ and the extended segment will still lie in $(-1,1)$. Again the factor $\epsilon$ does not depend on the position of $a$ and $b$, but only on $\chi(a, b)$.

PROOF OF THEOREM 2. We first show that $d$ convergence implies $\rho$ convergence for any convex $K$, whether symmetric or not. Let $P$ be a part of $K$ and let $x, y \in P, x \neq y$. Let $u_{n} \in E^{*},\left|u_{n}\right|<1$ on $K$, and $\chi\left(u_{n}(x), u_{n}(y)\right) \rightarrow \rho(x, y)>0$. Since eventually $u_{n}(x) \neq u_{n}(y)$, we may assume that $u_{n}(y)<u_{n}(x) \rightarrow a \leqq 0$, by taking a subsequence or replacing $u_{n}$ by $-u_{n}$ if necessary. Suppose that $[x, y]$ extends by $\epsilon$, so that $y+\epsilon(y-x) \in K$. The following identity is the result of an algebraic manipulation with the definition of $\chi$ :

$$
\begin{aligned}
1 & \geqq \chi\left(u_{n}(x), u_{n}(y+\epsilon(y-x))\right) \\
& =(1+\epsilon)\left[\chi\left(u_{n}(x), u_{n}(y)\right)^{-1}+\epsilon u_{n}(x)\right]^{-1} .
\end{aligned}
$$


Letting $n \rightarrow \infty$, we get

$$
\rho(x, y)^{-1}+\epsilon a \geqq(1+\epsilon), \quad \rho(x, y) \leqq[1+\epsilon(1-a)]^{-1} .
$$

Since $a \leqq 0$ and $\epsilon>0, \rho(x, y)<1$. If $d(x, y) \rightarrow 0$, so we may let $\epsilon \rightarrow \infty$, then it follows that $\rho(x, y) \rightarrow 0$. Hence the $d$ topology is stronger than the $\rho$ topology, and $x \sim y$ implies $\rho(x, y)<1$.

Now suppose that $K$ is symmetric, $P$ is a part, and $x, y \in P$. We know from the argument above that $\rho(x, y)<1$. Let $k=\rho(x, y)$, and let $\theta$ and $\epsilon$ be numbers such that $k+\theta<1$, and $\epsilon=\theta / k(1+k+\theta)$ as in Lemma 3. Let $u \in E^{*}$ and $|u|<1$ on $K$. Then $\chi(u(x), u(y)) \leqq \rho(x, y)$ $=k$. It follows that $[u(x), u(y)]$ extends by $\epsilon$ and the extended interval contains only points whose $\chi$-distance from $u(x)$ is less than $k+\theta$. Since $u$ is linear, $\chi(u(x), u(x+\epsilon(y-x)))<k+\theta<1$. In particular, $u(x+\epsilon(y-x)) \in(-1,1)$ whenever $u \in E^{*}$ and $|u|<1$ on $K$. This says that $x+\epsilon(y-x) \in K$ since $K$ is symmetric [6, p. 119]. Hence $[x, y]$ extends by $\epsilon$, and consequently $d(x, y) \leqq \log \left(1+\epsilon^{-1}\right)$. If $x, y \in K$ are arbitrary and $\rho(x, y)<1$, this argument still shows that $[x, y]$ extends by $\epsilon$. Thus $\rho(x, y)=k \rightarrow 0$ implies $\epsilon \rightarrow \infty$ and $d(x, y) \rightarrow 0$. Hence the $\rho$ topology is stronger and therefore equivalent if $K$ is symmetric; and $\rho(x, y)<1$ implies that $x \sim y$.

It is easy to see that if $K$ is not symmetric, then there can be points approaching the boundary of $K$ whose $\rho$ distances from a fixed point of $K$ would remain bounded. The intrinsic distances from a fixed point to points approaching the boundary necessarily approach $\infty$. This simply shows that the $\rho$ metric is not natural in a nonsymmetric setting. The intrinsic metric on the other hand does not depend on how $K$ is situated with respect to the origin, and in fact is invariant under translations of $K$.

\section{REFERENCES}

1. H. S. Bear, $A$ geometric characterization of Gleason parts, Proc. Amer. Math. Soc. 16 (1965), 407-412.

2. Errett Bishop, Representing measures for points in a uniform algebra, Bull. Amer. Math. Soc. 70 (1964), 121-122.

3. N. Bourbaki, Espaces vectoriels topologiques, Chapters I-II, Actualités Sci. Ind., No. 1189, Hermann, Paris, 1953.

4. A. M. Gleason, Function algebras, Seminar on analytic functions, Vol. 2, Institute for Advanced Study, Princeton, N. J., 1957.

5. E. Hille, Analytic function theory, Vol. 2, Ginn, Boston, Mass., 1962.

6. J. L. Kelley and Isaac Namioka, Linear topological spaces, Van Nostrand, Princeton, N. J., 1963.

7. Joseph Lewittes, $A$ note on parts and hyperbolic geometry, Proc. Amer. Math. Soc. 17 (1966), 1087-1090.

New Mexico State University and

University of California, Santa Barbara 